ENCYCLOPEDDIE Encyclopédie berbère

BERBERE

10 | 1991

10 | Beni Isguen - Bouzeis

\title{
Beraber (Linguistique)
}

\section{A. Willms}

\section{OpenEdition}

Journals

Édition électronique

URL : http://journals.openedition.org/encyclopedieberbere/1694

DOI : 10.4000/encyclopedieberbere.1694

ISSN : 2262-7197

\section{Éditeur}

Peeters Publishers

\section{Édition imprimée}

Date de publication : 1 décembre 1991

Pagination : 1473-1475

ISBN : 2-85744-549-0

ISSN : 1015-7344

\section{Référence électronique}

A. Willms, « Beraber (Linguistique)», Encyclopédie berbère [En ligne], 10 | 1991, document B66, mis en ligne le 01 mars 2013, consulté le 24 septembre 2020. URL : http://journals.openedition.org/ encyclopedieberbere/1694; DOI : https://doi.org/10.4000/encyclopedieberbere.1694

Ce document a été généré automatiquement le 24 septembre 2020

(C) Tous droits réservés 


\title{
Beraber (Linguistique)
}

\author{
A. Willms
}

1 La population berbérophone du Maroc se trouve essentiellement dans les zones montagneuses qui ont servi de refuge. Elle se partage en deux blocs : le bloc rifain au nord et le bloc formé par les Berabers et les Chleuhs du Maroc central et méridional. De ce fait le domaine des Berabers englobe le Moyen Atlas, les parties centrale et orientale du Haut-Atlas et le Djebel Sagho et ses environs. Les dialectes des Berabers sont désignés par les Berbères eux-mêmes sous le terme de tamaziyt.

2 Les différences entre ces grands blocs linguistiques, ainsi qu'à l'intérieur de chacun d'entre eux, sont graduelles et relativement faibles, bien qu'ici et là, l'histoire et les barrières géographiques aient contribué à créer des contrastes nets. L'action, de toutes façons faible, de l'espace en tant que facteur de différenciation dialectale a été réduite par certaines caractéristiques du berbère. Les variations morphologiques, que ce soit dans l'utilisation des éléments formels, leurs positions ou les transformations dues à des changements phonétiques, ont contribué à la constitution de parlers, elles ont aussi provoqué des divergences, parfois surprenantes, entre les idiolectes de personnes proches parentes.

3 André Basset alla plus loin en démontrant que les isoglosses du parler berbère ne convergent que trop rarement pour former de vraies frontières dialectales. De récentes études ont clairement prouvé que la différenciation dialectale de la langue berbère n'est qu'un reflet des nombreuses variations géographiques. Aussi n'est-il pas surprenant que l'on ne soit pas parvenu à une classification détaillée des parlers berbères marocains.

Dans ces conditions les idiomes des Berabers, sur un plan purement dialectologique, ne peuvent être considérés que comme un trait d'union entre le Chleuh du sud-ouest marocain, le Berbère rifain du nord marocain et le Berbère zenata d'Algérie. Les Chleuhs n'en conservent que des idiomes traditionnels du point de vue phonétique et morphologique, alors que le rifain révèle une série de décalages de phonèmes et que les idiomes zenata sortent du cadre en particulier à cause de modifications du schéma vocal. 
E. Destaing est à l'origine de la répartition devenue classique des dialectes berabers en dialectes septentrionaux du Moyen Atlas, méridionaux du Haut Atlas et du Djebel Sargho (E. Destaing, 1920). Il s'appuie pour ce faire sur « quelques particularités » de la phonétique, du vocabulaire et de la morphologie.

Des recherches récentes sur les dialectes des Aït Yafelman, des Aït 'Atta, des Aït Seddrat, des Ahel Todya et des Ahel Dades, ont montré que ces parlers conformément à leur situation géographique occupent du point de vue phonétique et du point de vue de l'inventaire des morphèmes, une position intermédiaire entre le chleuh, au sud-ouest, et les autres parlers berabers au nord. Ainsi, dans ces parlers berabers du sud, les phonèmes $/ t /$ et $/ d /$ ne sont pas, contrairement aux autres parlers berabers, réalisés comme $[t]$ et [d] mais, comme en chleuh, ou sous la forme [t] et [d]. Par contre, les phonèmes $/ \mathrm{k} /$ et $/ \mathrm{g} /$ sont la plupart du temps réalisés comme $[\mathrm{k}]$ et $[\overline{\mathrm{g}}]$, comme chez les autres Berabers, dans la mesure où chez ceux-ci, $[\mathrm{k}]$ et $[\overline{\mathrm{g}}]$ ne passent pas à $[\mathrm{c}]$ et $[\mathrm{j}]$, alors qu'en chleuh la spirantisation des phonèmes occlusifs est en général non attestée. Pourtant, parmi les Berabers du sud, les Ahel Todya et les Aït Seddrat suivent l'usage chleuh. Comme premier exemple relatif à l'inventaire des morphèmes nous signalerons que le mot chleuh /tifiyi/ (= viande) est aussi employé par une partie des Berabers du sud, cependant le mot /aksum/ est plus généralement employé chez les Berabers du sud qui se conforment ainsi à l'usage des autres Berabers. Notre deuxième exemple est l'utilisation, certes très rare, de la particule du futur chleuh / rad/ par des Berabers du sud originaires de différents lieux, preuve que le problème abordé ici de la diffusion de certaines particularités dialectales se pose aussi pour les morphèmes non libres.

\section{BIBLIOGRAPHIE}

Abdel-Massih E., A course in spoken Tamazight : Berber dialects of Ayt Ayache and Ayt Seghrouchen (Middle Atlas), Morocco, Ann Arbor, 1971.

Id., A reference grammar of Tamazight : a comparative study of the Berber dialects of Ayt Ayache and Ayt Seghrouchen (Middle Atlas), Morocco, Ann Arbor, 1971.

Id., Tamazight Verb structure : a generative approach, Doctoral diss., Ann Arbor, 1968, The Hague, 1971.

Abes, Première année de langue berbère (dialecte du Maroc central), Rabat, 1916.

Basset A., « Notules Berbères Aït Sadden », Groupe linguistique d'études chamito-sémitiques (GLECS), 7, pp. 16-18.

$I d$., « Sur le berbère Aït Sadden it $(\mathrm{t}) \mathrm{h}$ "parce que" et la formation du système conjonctif », Groupe linguistique d'études chamito-sémitiques (GLECS), 6, p. 64.

Id., « Sur le participe dans le parler berbère des Aït Sadden », Journal asiatique, 1954, t. 242, pp. 393-395.

Id., Textes berbères du Maroc (parler des Aït Sadden), Paris, 1963. 
Biarnay S., « Notice sur les dialectes berbères parlés par les Aïth Sadden et les Beni Mgild », Revue Africaine, 1911, t. 55, pp. 200-245.

Id., « Six textes en dialecte berbère des Beraber de Dadès », Journal asiatique, 1912, pp. 347-371. Bisson J., Leçon de berbère tamazight, Rabat, 1940.

Boulifa ('Ammār Ibn-Sa'īd), Textes berbères en dialecte de l'Atlas marocain, Paris, 1909.

Bynon J., « Berber nursery language », Transaction of the Philological Society, 1968, pp. 107-161.

Id., « A class of Phonaesthetic words in Berber », African Language studies, 1970, vol. 9, pp. 64-80.

Id., « The Derivational processes relating berber nursery words to their counterparts in normal Inter-Adult speech », in Talking to children, Cambridge, 1977, pp. 255-263. Id., « Riddle telling among the Berbers of central Morocco », African language studies, 1966, vol. 7, pp. 80-104, 1967, vol. 8, pp. 168-197.

Destaing E., Étude sur le dialecte berbère des Aït Seghrouchen, 1920.

Drouin J., «A propos de quelques noms de tissu dans un village berbérophone du Maroc central », Groupe linguistique d'études chamito-sémitiques (GLECS), 12 13, pp. 135-147.

Galand L., « 1. y ancien et $\mathrm{y}<\mathrm{q}$ dans le parler berbère des Aït Sadden (Maroc). 2. Un cas d'opposition pertinente w/u en berbère ", Groupe linguistique d'études chamito-sémitiques (GLECS), 7, pp. 91-92.

Galand J, « Observations sur les emplois de deux emprunts du berbère (Ait Youssi d'Enjil) à l'arabe : 1. tlata "trois". 2. kuls » i “tout, tous" », Groupe linguistique d'études chamito-sémitiques (GLECS), 9, (1.) : pp. 68-70; (2.) : pp. 71-74.

Galand-Pernet P., « Remarques sur la langue de la version berbère de Tinrhir (Maroc) de la Haggâdâh de Pesah », Groupe linguistique d'études chamito-sémitiques (GLECS), 12-13, pp. 168-181.

Galand-Pernet P. et Zafrani H., Une version berbère de la Haggâdâh de Pesah : texte de Tinrhir du Todrha (Maroc), Paris, 1970, 2 vol.

Harries J., « Verbless sentences and "verbs ofBeing" in Tamazight », Papers in African Linguistics, 1971, pp. 113-121.

Johnson M., Syntactic structures of Tamazight, Doctoral diss., Los Angeles, 1966.

Kaoui S. Cid, Dictionnaire français-tachelh'it et tamazir't (dialectes berbères du Maroc), Paris, 1907.

Laoust E., " Chants berbères contre l'occupation française ", in Mémorial Henri Basset, Paris, 1928, t. 2, pp. 9-20.

Id., « Contes berbères du Maroc. Textes berbères du groupe beraber-chleuh (Maroc central, Haut et Anti-Atlas) », Publications de l'Institut des hautes études marocaines, t. 50, Paris, 1949.

Id., Cours de berbère marocain, dialecte du Maroc central, $2^{\mathrm{e}}$ éd., Paris, 1928.

Id., Étude sur le dialecte berbère des Ntifa, Paris, 1918.

Id., Mots et choses berbères, Paris, 1920.

Id., « Un texte dans le parler berbère des Aït Messad », in 'Mélanges René Basset, Paris, 1925, t. 2, pp. 305-334.

Loubignac V., Etude sur le dialecte berbère des Zaïan et Ait Sgougou, Paris, 1924, 2 vol.

Mercier H., Vocabulaires et textes berbères dans le dialecte des Aït Izdeg, Rabet, 1937. 
Pellat Ch., « Deux textes dans le parler berbère des Aït bu Zeggu de Mestigmeur », Revue Africaine, 1947 , t. 91 , pp. 254-259.

Pellat Ch., « Notes de géographie linguistique (berbère marocain) », Communications et rapports, $\mathrm{n}^{\circ}$ 193, pp. 131-150.

Penchoen J., Tamazight of the Ayt Ndhir, Los Angeles, 1973.

Prasse K., " Les relations de sexe, d'âge et de sang : enquête sur la terminologie dans le dialecte berbère des Ayt-Sadden ", Acta orientalis, 1957, t. 22, pp. 118-141.

Reyniers F., Taougrat ou les Berbères racontés par eux-mêmes, Paris, 1930.

Roux A., « Un chant d'amdyaz, l'aède berbère du groupe linguistique beraber ", in Mémorial Henri Basset, Paris, 1928, t. 1, pp. 237-242.

Id., Récits, contes et légendes dans le parler des Beni Mtir, Rabat, 1942.

Id., «Le verbe dans le parler berbère des Ighezran, Beni Alahm et Marmoucha », Bulletin de la société linguistique de Paris, 1935, t. 36, pp. 43-78.

Serra L., « In margine a un testo orale berbero avente a titolo "La storia della gente di Sigil-massa "», Studi magrebini, 1974, 6, pp. 57-71.

Willms A., « Grammatik der Südlichen Beraberdialekte (Südmarokko) », Afrikanistische Forschungen, Bd 6, Glückstadt, 1972.

Id., « Sekundäre Kontrastierung in Ergänzung der Konsonantenlänge im Berberischen Südmarokkos », Afrika und Ubersee, Bd 48, 1964, pp. 289-293.

INDEX

Mots-clés : Linguistique, Maroc, Tribus 